\title{
Centro de pesquisa universitária: caracterização do ambiente de pesquisa
} \author{
ROQUE RABECHINI JUNIOR ${ }^{2}$ \\ ${ }^{1}$ UNIVERSIDADE DO ESTADO dO RIO DE JANEIRO (UERJ), RIO DE JANEIRO - RJ, BRASIL \\ ${ }^{2}$ Universidade nove de Julho (UninOVE) / Programa de Pós-graduação em Gestão de Projetos, SÃo Paulo - SP, Brasil
}

JosÉ DA ASSUNÇÃO MOUTINHO ${ }^{12}$

\begin{abstract}
Resumo
As possibilidades de estreitamento das relações entre universidades e empresas por meio dos Centros de Pesquisa Universitária têm gerado oportunidades de desenvolvimento de conhecimento e avanço tecnológico entre outras vantagens. Entender as relações existentes entre estes atores, certamente, produzirá melhorias no desenvolvimento tecnológico e social que permeiam esse ambiente. Este artigo visa compreender como se caracteriza o ambiente colaborativo formado por CPUs, Universidades e Empresas. Como estratégia de pesquisa, optou-se pela revisão sistemática da literatura que, a partir das buscas nas bases Scopus e Web of Science, resultou em 63 artigos que compuseram a amostra final. Como resultado, descreve as características de CPUs, motivações dos atores para se estabelecer relacionamentos, principais modalidades e formas de colaboração, medição de resultados da colaboração, performance dos CPUs, assim como transferências de conhecimento e tecnologia entre os colaboradores. A pesquisa também revela que a teoria do capital humano, a visão baseada em recursos, a teoria institucional e a lógica institucional são as principais lentes teóricas utilizadas nos estudos analisados. Por fim, apresenta-se um framework que integra os elementos discutidos.
\end{abstract}

Palavras-chave: Centros de pesquisa. Teorias. Pesquisa universitária.

\section{University research centers: research environment characterization}

\begin{abstract}
The possibilities for closer relations between universities and companies through University Research Centers (URCs) have created opportunities for knowledge development and technological advancement, among other advantages. Understanding existing relationships between these actors improve technological and social development of this environment. This paper aims to understand how the collaborative environment formed by URCs, Universities, and Companies is characterized. Through a systematic literature review, based on searches in the Scopus and Web of Science, 63 articles made up the final sample. The results show the characteristics of URCs, motivations of the actors to establish relationships, main modalities and forms of collaboration, measurement of results of collaboration, performance of UCRs, and knowledge and technology transfers among employees. The research reveals that the human capital theory, the resource-based view, the institutional theory, and the institutional logic are the main theoretical lenses used in the analyzed studies. Finally, we present a framework that integrates the discussed elements.
\end{abstract}

Keywords: Research Centers. Theories. University research.

\section{Entorno colaborativo en centros universitarios de investigación: caracterización del entorno de investigación}

\section{Resumen}

Las posibilidades de relaciones más estrechas entre universidades y empresas a través de los Centros de Investigación Universitaria (CIU) han generado oportunidades para el desarrollo del conocimiento y el avance tecnológico, entre otras ventajas. Comprender las relaciones existentes entre estos actores ciertamente producirá mejoras en el desarrollo tecnológico y social que impregna este entorno. Este artículo tiene como objetivo comprender cómo se caracteriza el entorno de colaboración formado por CIU, universidades y empresas. Como estrategia de investigación, se eligió la revisión sistemática de la literatura que, con base en búsquedas en las bases de datos Scopus y Web of Science, resultó en 63 artículos que conformaron la muestra final. Como resultado, describe las características de los CIU, las motivaciones de los actores para establecer relaciones, las principales modalidades y formas de colaboración, la medición de los resultados de la colaboración, el rendimiento de los CIU, así como las transferencias de conocimiento y tecnología entre los colaboradores. La investigación también revela que la teoría del capital humano, la visión basada en los recursos, la teoría institucional y la lógica institucional son las principales lentes teóricas utilizadas en los estudios analizados. Finalmente, se presenta un marco que integra los elementos discutidos.

Palabras clave: Entornos colaborativos. Teorías. Investigación universitaria. 


\section{INTRODUÇÃO}

As interações entre universidades, empresas e governos se tornam cada vez mais intensas e importantes no contexto socioeconômico. A academia busca uma nova definição para o seu papel aproximando-se da sociedade e das empresas. As empresas necessitam reciclar suas alternativas de competitividade visando garantir a permanência no mercado. $O$ governo precisa desenvolver políticas públicas beneficiando a sociedade e garantir o círculo virtuoso. De fato, universidades, empresas e governo precisam encontrar formas de se relacionar. Os centros de pesquisa universitária (CPUs) podem colaborar para o preenchimento desta lacuna enquanto agentes integradores (Youtie, Libaers \& Bozeman, 2006).

O formato atual dos CPUs, que representa um tipo de pesquisa colaborativa, origina-se nos Estados Unidos, na experiência entre cientistas e engenheiros em projetos conjuntos durante a Segunda Guerra Mundial. As colaborações bem-sucedidas chamaram a atenção de pesquisadores renomados sobre a forma mais produtiva de se desenvolver pesquisas científicas, tendo servido como forte impulso para a formação de diversos outros CPUs no período pós-guerra (Etzkowitz \& Kemelgor, 1998). Ao longo dos anos, os CPUs cresceram em número, em grande parte pelo fato de os formuladores de políticas acreditarem que iniciativas de pesquisa em maior escala, baseadas em equipe, multidisciplinares, estrategicamente focadas e multifacetadas são mais propensas a produzir pesquisa de alto valor e de alto impacto quando comparadas com as formas tradicionais de investigação individual (Gray, 2008).

Os CPUs desempenham um papel fundamental na promoção do crescimento do conhecimento. São também responsáveis por incentivar a construção de redes colaborativas e pela promoção do capital humano científico e técnico, unindo pesquisadores de origens variadas, com o objetivo de endereçarem problemas cada vez mais complexos (Berbegal-Mirabent, Sánchez García \& Ribeiro-Soriano, 2015; Bozeman \& Boardman, 2003). A definição e o entendimento iterativo bidirecional entre CPUs e os ambientes externos é vital para incrementar a tradução bem-sucedida da pesquisa universitária.

De fato, a aproximação entre universidade e empresas traz novas perspectivas. Pelo lado das universidades, os pesquisadores são incentivados a desenvolverem seus estudos, linhas mais pragmáticas, indo além das fronteiras científicas fundamentais (Sabharwal \& Hu, 2013). Para as empresas e governos, trata-se de um novo parceiro para seus esforços de pesquisa e desenvolvimento, permitindo-Ihes ter acesso a recursos de acadêmicos, incluindo instalações e pessoal altamente qualificado (Bruneel, D'este \& Salter, 2010).

As colaborações estabelecidas entre universidades e empresas por intermédio dos CPUs têm sido cada vez mais reconhecidas como mecanismos para oportunidades de criação de conhecimento e troca de tecnologia entre atores, levando a efeitos sinérgicos e possibilidades de círculos virtuosos (Geuna \& Muscio, 2009). Seus inúmeros tipos de resultados se materializam e aproximam estudantes, pesquisadores, gestores e profissionais (Rogers et al., 1999) e definem o perfil dos CPUs (Corona, 2015; Stahler \& Tash, 1994).

A breve contextualização sobre CPUs mostra a relevância do tema que extrapola fronteiras do ambiente universitário. A literatura visitada por um lado parece fragmentada (Galán-Muros \& Davey, 2017), mas por outro abre um leque de possibilidade a explorar, remetendo à questão de pesquisa: Quais são os principais elementos presentes na literatura que formam o ambiente colaborativo definido entre Centros de Pesquisa Universitária, Universidades e Empresas? Consequentemente, o objetivo da pesquisa é compreender como se caracteriza o ambiente colaborativo formado por CPUs, Universidades e Empresas.

Para alcançar o objetivo proposto, os autores recorreram à Revisão Sistemática da Literatura (RSL) como estratégia, nas bases Scopus e Web of Science, que foi incrementada com o uso da técnica denominada snowballing. Para serem incluídos na amostra os artigos deveriam atender a um ou mais dos seguintes critérios: descrever as características de CPUs, motivações dos atores para estabelecer relacionamentos, principais modalidades e formas de colaboração, medição de resultados da colaboração, performance dos CPUs, assim como transferências de conhecimento e tecnologia entre os colaboradores. A pesquisa também investigou as principais lentes teóricas usadas nos artigos considerados, revelando ser a teoria do capital humano, a visão baseada em recursos, a teoria institucional e a lógica institucional. Por fim, o estudo apresenta um framework que integra os elementos discutidos. 


\section{PROCEDIMENTO METODOLÓGICO}

Amplamente utilizado na área de saúde (Sackett, Rosenberg, Gray, Haynes \& Richardson, 1996), a RSL vem ganhando espaço para sua aplicação na área de Ciências Sociais (Petticrew \& Roberts, 2006; Tranfield, Denyer \& Smart, 2003). A RSL é conduzida por meio de um processo composto por uma sequência de fases predefinidas, sendo uma forma rigorosa, confiável e passível de auditoria, reduzindo vieses subjetivos e riscos de negligenciar a literatura relevante (Brereton, Kitchenham, Budgen, Turner \& Khalil, 2007).

O primeiro passo da RSL consistiu na definição da string de busca e demais parâmetros. As expressões gerais utilizadas foram (universit* OR academ * OR scientific* OR "higher education") AND (research OR investigation) AND (cent* OR lab* OR core*) pesquisada no campo título, sendo considerados apenas os artigos de periódicos revisados por pares. Somente trabalhos publicados no idioma inglês foram incluídos além das áreas business, "education educational research" e management. As bases escolhidas foram ISI Web of Science (WOS) e Scopus por congregarem as revistas de maior impacto da área de gestão. Não houve restrição em relação aos anos de publicação dos artigos, sendo as buscas nas bases realizadas em maio 2020.

Como critérios de inclusão na amostra, foram considerados apenas estudos primários e que tratassem de caracterização de CPUs, motivações para se estabelecer relacionamento com CPUs, principais modalidades e formas de colaboração com CPUs, medição de resultados da colaboração, performance dos CPUs, assim como transferências de conhecimento e tecnologia entre os colaboradores. Os critérios de exclusão da amostra final levaram em consideração: artigos publicados apenas como resumo, ou não escritos em inglês, ou versão mais antiga de um outro estudo já considerado na amostra, ou estudo não primário, ou estudo sem condição de acesso integral.

O processo de decisão pela inclusão ou não dos artigos contou com a participação ativa dos dois autores. Este procedimento visou reduzir probabilidade de descartar estudos relevantes (Edwards et al., 2002). Em termos de configuração, um pesquisador realizou a seleção dos trabalhos e o segundo revisou a decisão de incluir ou não na amostra, seguindo as recomendações de Felizardo, Nakagawa, Fabbri e Ferrari (2017), o que resultou na Tabela 1.

Visando ampliar a base bibliográfica consultada, os autores colocaram em prática a estratégia secundária denominada snowballing (Wohlin, 2014) que, assim como a RSL, buscou estudos primários. Petersen, Vakkalanka e Kuzniarz (2015) afirmam que se pode obter resultados mais consistentes ao se combinar as duas estratégicas (RSL e snowballing), já que a obtenção de estudos primários apenas com uma RSL pode trazer limitações relacionadas aos parâmetros da string. Assim, os autores aplicaram a técnica para cada um dos 35 artigos que compuseram a amostra inicial. 0 processo resultou em 26 novos estudos no processo reverso e 2 novos no processo avante, totalizando 63 artigos.

Tabela 1

Composição da amostra inicial

\begin{tabular}{lcccc}
\hline Fonte & $\begin{array}{c}\text { Incluídos na } \\
\text { amostra }\end{array}$ & $\begin{array}{c}\text { Excluídos } \\
\text { da amostra }\end{array}$ & $\begin{array}{c}\text { Artigo } \\
\text { inacessível }\end{array}$ & Total \\
\hline Wos & 7 & 71 & 8 & $\mathbf{8 6}$ \\
\hline Scopus & 13 & 52 & 1 & $\mathbf{6 6}$ \\
\hline Ambas as bases & 15 & 26 & 0 & $\mathbf{4 1}$ \\
\hline Total & $\mathbf{3 5}$ & $\mathbf{1 4 9}$ & $\mathbf{9}$ & $\mathbf{1 9 3}$ \\
\hline
\end{tabular}

Fonte: Elaborada pelos autores.

Uma vez definida a amostra e organizados os metadados, cada artigo foi analisado, visando trazer subsídios para formação do ambiente colaborativo em CPU, à luz de cada um dos critérios de inclusão. Foram extraídos dados gerais e específicos de cada estudo primário, sendo mapeados em planilha eletrônica específica. O processo de síntese dos dados extraídos contou com dois métodos. $\mathrm{O}$ integrativo, sumarizando e agrupando evidências, e o interpretativo, organizando conceitos identificados como abrangentes. 


\section{APRESENTAÇÃO E DISCUSSÃo DOS RESULTADOS}

Para facilitar a organização da RSL, a seção de resultados foi dividida seguindo os critérios de inclusão dos artigos. Na sequência, foram identificadas as principais teorias utilizadas e, por fim, apresentada a proposta do framework.

\section{Caracterização de centros de pesquisa}

Não há consenso na literatura sobre a definição precisa de um centro de pesquisa, devido à heterogeneidade e à ampla diversidade de objetivos, variando substancialmente em termos de suas características organizacionais (Etzkowitz \& Kemelgor, 1998; Stahler \& Tash, 1994). Como ponto comum a todos, há intenção de promover algum grau de colaboração entre pesquisadores. A composição dessas colaborações pode ser multidisciplinar, interorganizacional, intersetorial, de acordo com objetivos científicos e técnicos do centro (Bozeman \& Boardman, 2003; Rogers et al., 1999). Existem centros que são criados especificamente para induzir interações e colaborações entre pesquisadores em vários departamentos e disciplinas, universidades, indústria, governo e, por vezes, em vários países (Lal, Boardman, Towery, Link \& Shipp, 2007).

A definição de centro de pesquisa proposta por Boardman e Gray (2010) tem sido adotada em diversos trabalhos. Trata-se de uma organização ou unidade dentro de uma organização maior que realiza pesquisas e possui missão explícita (e atividades relacionadas) para promover, direta ou indiretamente, a colaboração intersetorial, transferência de conhecimento (TC) e de tecnologia (TT) e, finalmente, inovação.

Bozeman e Boardman (2003) definem CPU como entidade organizacional formal dentro de uma universidade que existe com a missão específica de servir à pesquisa, distinta do departamento acadêmico e que pode incluir pesquisadores de mais de um departamento. Nessa definição, percebe-se que não há exigência que um CPU seja interdisciplinar ou multidisciplinar. Os autores chamam atenção para a distinção que talvez seja a mais importante: os CPUs desenvolvem interações com stakeholders externos à universidade, incluindo outras universidades, empresas, indústria e governo. Sua criação ocupa lacunas entre universidades e empresas que não foram preenchidas pela própria universidade, seus laboratórios e departamentos acadêmicos e institutos de pesquisa (Ponomariov \& Boardman, 2010; Styhre \& Lind, 2010), e que são gerenciados separadamente das atividades acadêmicas normais (Sá, 2008).

CPUs são percebidos como mecanismos específicos pelos quais empresa e universidades criam pontes organizacionais que ultrapassam os limites das diferenças culturais e estruturais (Nursall, 2003). Recursos e infraestrutura podem vir da universidade, mas geralmente se desenvolvem e se consolidam com subsídios externos graças ao esforço dos seus pesquisadores (Torres Zapata, 2019). Outro fator emergente nesse sistema é a formação de alianças. Essas alianças entre universidades e instituições públicas e privadas mudaram a maneira como a estrutura organizacional dos CPUs é definida (Magro \& Wilson, 2013).

A literatura também apresenta diversas taxonomias em função da iniciativa de sua criação, afiliação de seus pesquisadores e ainda dos acordos cooperativos. Bozeman e Boardman (2013) classificam em Centro de Pesquisa Universitária, Centro de Pesquisa do Estado e Centro de Pesquisas de Agências Federais. Já Santoro e Chakrabarti (2001) classificam em Centros de Pesquisa de Engenharia, Centros de Pesquisa Cooperativa Universidade-Indústria e Centros de Pesquisa Universitária. É fato que os CPUs representam a maioria dos centros de pesquisa. Mesmo assim, a literatura tem dedicado menos atenção a esse tipo de centros, não obstante seu domínio quantitativo. Estudos baseados em dados de CPUs enfatizaram diferenças com os demais tipos anteriormente citados (Boardman, 2009; Boardman \& Corley, 2008), mas não abordaram profundamente esse tipo dominante.

Considerando a pluralidade de atividades, os CPUs tendem a ter portfólios de pesquisa heterogêneos e a apresentar maiores desafios gerenciais quando comparados às atividades acadêmicas tradicionais. Tais desafios requerem conhecimento de abordagens mais estruturadas de gestão por parte líderes (pesquisadores principais) para organização e gestão dos CPUs (Boardman \& Ponomariov, 2012), e ainda por lidarem com uma ampla variedade de pesquisadores envolvidos com empresas industriais (Gaughan \& Corley, 2010). Em geral, os impactos dos CPUs tendem a ser difusos, abrangendo além dos resultados acadêmicos, comerciais e de alcance social (Bozeman \& Gaughan, 2007; Gray, 2000). 


\section{Motivação para se estabelecer relacionamento com CPUs}

Problemas científicos relevantes exigem múltiplas competências e a integração de diversas perspectivas disciplinares (Carayannis, Del Giudice \& Della Peruta, 2014). Principalmente a partir da Primeira Guerra Mundial, as colaborações entre mercado e academia cresceram a passos largos, estreitando laços por várias razões (Geiger, 1990). Os motivos pelos quais os diferentes atores, como universidades, empresas e governo, participam de colaborações têm sido objeto de diversas pesquisas ao longo de décadas. O Quadro 1 apresenta as principais motivações identificadas na literatura consultada.

Como se pode verificar no Quadro 1, algumas motivações têm caráter específico enquanto outras são mais gerais. Na prática, atores envolvidos podem identificar múltiplas motivações. Além disso, motivações das partes envolvidas devem ser observadas, visto que uma incompatibilidade pode levar a conflitos, colocando em jogo frutos da colaboração. Essas motivações representam valores que os membros percebem ao criar e participar dessas colaborações (Horne, Poulin \& Frayret, 2010).

\section{Quadro 1 \\ Motivações dos atores}

\begin{tabular}{|c|l|}
\hline Atores & \multicolumn{1}{c|}{ Motivações } \\
\hline Universidades & $\begin{array}{l}\text { Aportes financeiros para estudantes; patentes; contratos de licença; criação de spin-off; desenvolvimento de } \\
\text { novos produtos; respostas às políticas do Governo; oportunidades de emprego para estudantes de pós-graduação; } \\
\text { publicação de trabalhos; acesso a problemas relevantes de pesquisa; melhoria na implementação de novas } \\
\text { tecnologias; acesso a recursos adicionais; insight sobre próprias pesquisas; aplicação de pesquisas em problemas } \\
\text { reais; aumento do poder estratégico; contribuição para políticas de Governo; futuras oportunidades de negócios; } \\
\text { possibilidade de pesquisa de vanguarda; acesso a empresas para pesquisa aplicada; integração teoria-prática; } \\
\text { acesso a redes de pesquisa; acesso aos mercados protegidos; e criação de vínculos com empresas. }\end{array}$ \\
\hline Empresas & $\begin{array}{l}\text { Acesso a pessoal altamente qualificado; acesso a treinamento e suporte para desenvolvimento de habilidades } \\
\text { internas; resolução de problemas específicos; acesso a instalações universitárias; acesso a financiamento para } \\
\text { P\&D; comercialização de tecnologias baseadas na universidade; acesso a variedade de instalações de treinamento } \\
\text { pós-experiência que ajudou a projetar; acesso à pesquisa, consultoria e coleta de dados da universidade; acesso a } \\
\text { estado-da-arte científico; intercâmbio de conhecimento entre universidade-empresa; compartilhamento de riscos; } \\
\text { diminuição de custos; melhoria das imagens interna e externa e a reputação da empresa; ampliação do escopo } \\
\text { de atividades; criação de oportunidades de investimentos; acesso a redes de pesquisa; aumenta a capacidade } \\
\text { absortiva; aumenta a quantidade de estudantes talentosos atraídos; procura por novos produtos e novas tecnologias; } \\
\text { e, aquisição de tecnologias antes não disponíveis. }\end{array}$ \\
$\begin{array}{l}\text { Aumento do emprego; aumento da capacidade absortiva por parte de empresas; impulsiona o sistema de inovação; } \\
\text { aumenta produtividade das empresas e a geração de riqueza; corrige falhas de mercado no investimento em P\&D; } \\
\text { acelera a inovação tecnológica; cria redes de pesquisa; aumenta o intercâmbio de informaçães entre universidades } \\
\text { e empresas; e, gera desenvolvimento econômico, social e cultural. }\end{array}$ \\
\hline Governo
\end{tabular}

Fonte: Adaptado de Ahn (1995), Hayton, Sehili e Searpello (2010), Lee (2000), Lee e Win (2004), Motohashi (2005), Phillips (1991), Santoro e Chakrabarti (1999, 2001) e Styhre e Lind (2010).

\section{Principais modalidades e formas de colaboração}

A literatura indica diversas modalidades de colaboração (Boardman, 2009; Bruneel et al., 2010). Suas formas de implementação variam de acordo com o grau com que os partícipes se integram (Boardman \& Corley, 2008). As relações se diferenciam de acordo com o "nível" das interações entre os atores, que podem ser de nível individual, institucional ou entre os dois níveis e por meio das modalidades de interações pelas quais é realizada como no caso de um conhecimento codificado (como uma publicação científica), um artefato tecnológico (como um protótipo) ou mesmo um fluxo financeiro (como uma transferência de licença). O ponto comum a qualquer tipo de colaboração gira em torno do aumento do estoque de conhecimento de pelo menos um dos colaboradores (Inzelt, 2004).

Também é necessário diferenciar o sentido da interação. Por um lado, é possível observar interações unilaterais do CPU com empresas, como a prestação de serviços de P\&D realizados por equipes de pesquisa universitária para empresas. Mas também há interações bilaterais através das quais há uma troca real de conhecimento entre os parceiros, conhecido como two-way bridge (Meyer-Krahmer \& Schmoch, 1998). Os termos da colaboração entre CPUs e empresas não podem, portanto, ser vistos todos no mesmo nível (Tidd \& Trewhella, 1997), sendo ainda vulneráveis a conflitos (Tidd \& Izumimoto, 2002). 
É possível identificar cinco dimensões para caracterizar as possíveis formas de colaboração (Quadro 2). O sucesso depende, em última análise, da capacidade dos parceiros de criar confiança (Le Roy, Robert \& Lasch, 2016), comprometimento e benefícios mútuos (Numprasertchai \& Igel, 2005).

\section{Quadro 2}

Principais Modalidades de Colaboração

\begin{tabular}{|c|c|c|c|c|c|}
\hline Tipo de colaboração & $\begin{array}{l}\text { Níveis de } \\
\text { interação }\end{array}$ & $\begin{array}{c}\text { Grau de } \\
\text { formalização }\end{array}$ & $\begin{array}{l}\text { Conhecimento } \\
\text { científico registrado }\end{array}$ & $\begin{array}{l}\text { Artefato } \\
\text { tecnológico }\end{array}$ & $\begin{array}{c}\text { Fluxo } \\
\text { financeiro }\end{array}$ \\
\hline Joint-venture & Institutional & Alto & Pode haver & Pode haver & Sim \\
\hline $\begin{array}{l}\text { Projeto de pesquisa } \\
\text { desenvolvido em parceria }\end{array}$ & Institutional & Alto & Pode haver & Pode haver & Pode haver \\
\hline Contrato de pesquisa & Institutional & Alto & Pode haver & Pode haver & Sim \\
\hline Prestação de serviços em P\&D & $\begin{array}{l}\text { Individual e } \\
\text { institutional }\end{array}$ & Alto & Não & Pode haver & Sim \\
\hline $\begin{array}{l}\text { Contratação de empresas por } \\
\text { parte dos CPUs }\end{array}$ & Institutional & Alto & Sim & Pode haver & Sim \\
\hline $\begin{array}{l}\text { Acesso a instalações e } \\
\text { equipamentos das empresas }\end{array}$ & $\begin{array}{l}\text { Individual e } \\
\text { institutional }\end{array}$ & Baixo & Não & Sim & Pode haver \\
\hline $\begin{array}{l}\text { Acesso a instalações e } \\
\text { equipamentos dos CPUs }\end{array}$ & $\begin{array}{l}\text { Individual e } \\
\text { institutional }\end{array}$ & Baixo & Não & Sim & Pode haver \\
\hline $\begin{array}{l}\text { Contatos informais entre } \\
\text { acadêmicos e funcionários de } \\
\text { empresas }\end{array}$ & Institutional & Baixo & Pode haver & Pode haver & Não \\
\hline Spin-off & $\begin{array}{l}\text { Individual e } \\
\text { institutional }\end{array}$ & Médio & Pode haver & Pode haver & Sim \\
\hline
\end{tabular}

Fonte: Adaptado de Boardman (2009), Boardman e Corley (2008), Bruneel et al. (2010), Inzelt (2004), Meyer-Krahmer e Schmoch (1998) e Scott, Steyn, Geuna, Brusoni e Steinmuller (2001).

As diferentes configurações de colaboração entre CPUs e empresas, identificadas na literatura, geram resultados distintos. É possível citar, por exemplo, aumento do estoque de conhecimento das partes envolvidas, melhoria e desenvolvimento de metodologias, novas patentes e registro, treinamento de pesquisadores em potencial e desenvolvimento de habilidades e competências, criação e desenvolvimento de redes de colaboração, resolução de problemas técnicos específicos que surgem com as empresas além de nascimentos de novos negócios como spin-off universitário (Boardman \& Corley, 2008; Rogers et al., 1999).

\section{Medição dos resultados da colaboração}

Como se pôde verificar nas seções anteriores, a colaboração entre CPUs e empresas abre oportunidade para gerar contribuições importantes para ambos os atores. No entanto, na prática, esses potenciais benefícios nem sempre se materializam (GalánMuros \& Plewa, 2016) devido a objetivos conflitantes das partes envolvidas. Essas diferenças podem ser atribuídas a uma dicotomia entre lógicas divergentes entre os atores. No entanto, há evidências que a proximidade sociocognitiva entre CPU-E é fundamental para o alcance dos resultados da colaboração (Lauvås \& Steinmo, 2019).

A sustentabilidade dessa experiência colaborativa, concentra-se nos resultados reais de "dar e receber" entre pesquisadores das universidades e empresas. O benefício mais significativo obtido pela empresa é o aumento do acesso a novas pesquisas e descobertas universitárias. Pelo lado da universidade, os pesquisadores estão complementando sua própria pesquisa acadêmica, garantindo fundos para estudantes e equipamentos, e ampliando o acesso a informações sobre suas próprias pesquisas. Ainda que possa acarretar uma sobrecarga de trabalho, face aos compromissos acadêmicos que desempenham em departamentos universitários (Boardman \& Bozeman, 2007), as experiências colaborativas tendem a se sustentar ou mesmo se expandir (Lee, 2000). 
Medir a eficácia do relacionamento CPU-E não é uma questão trivial (Gray \& Steenhuis, 2003). A pesquisa conduzida por Santoro e Chakrabarti (1999) leva em conta três dimensões: entrada de recursos no centro universitário, participação no processo de relacionamento CPU-E, e os resultados do relacionamento. A transferência explícita capitaliza as atividades de relacionamento de CPU-E para integrar a pesquisa baseada na universidade para impulsionar iniciativas aplicadas ao desenvolvimento e comercialização de novas tecnologias. Pode ser medido em termos de patentes, licenças, publicações e uso conjunto de instalações e equipamentos (Evans, Starbuck, Kiresuk \& Gee, 1993).

\section{Medição dos resultados do CPU}

CPUs requerem perspectivas de longo prazo e objetivos claros (Geisler, Furino \& Kiresuk, 1990). Apesar das crescentes demandas para avaliar o desempenho de CPUs, existem poucos quadros e métodos teóricos para fazê-lo. A eficácia de um centro bem-sucedido depende, até certo ponto, da avaliação do progresso de seus programas em andamento, a fim de capturar as suas realizações (Lee, Kim \& Cha, 2014; Nishimura, Nishimura, Goebert, Onoye \& Sugimoto-Matsuda, 2018), assim como da intensidade de participação dos pesquisadores (Kassab, Mutz \& Daniel, 2019) e gestores (Gray, 2008).

Os estudos de Boardman e Gray (2010) e Roessner, Manrique e Park (2010) mostraram evidências de lacunas nos processos de avaliação de desempenho de CPUs dada a complexidade do ecossistema, falta de dados e inadequação dos indicadores tradicionais. A estrutura, os processos e a moral dos membros tendem a variar de acordo com a idade do CPU, o que influencia consequentemente a variação no desempenho organizacional. De fato, não é óbvio quais padrões e critérios de medição devem ser usados para avaliar a eficácia dos CPUs (Cameron \& Whetten, 1981).

A capacidade de alcançar os objetivos dos CPUs depende da definição de sistema de avaliação transparentes para auxiliar na tomada de decisões. Gibson, Daimb e Dabic (2019) propõem um framework hierárquico de decisão utilizando parâmetros quali-quantitativos (Figura 1). Trata-se de um modelo que leva em consideração a missão, os objetivos, as metas e os resultados do CPU. O modelo é generalizável e pode ser aplicado para avaliação de desempenho entre CPUs.

Figura 1

Estrutura hierárquica

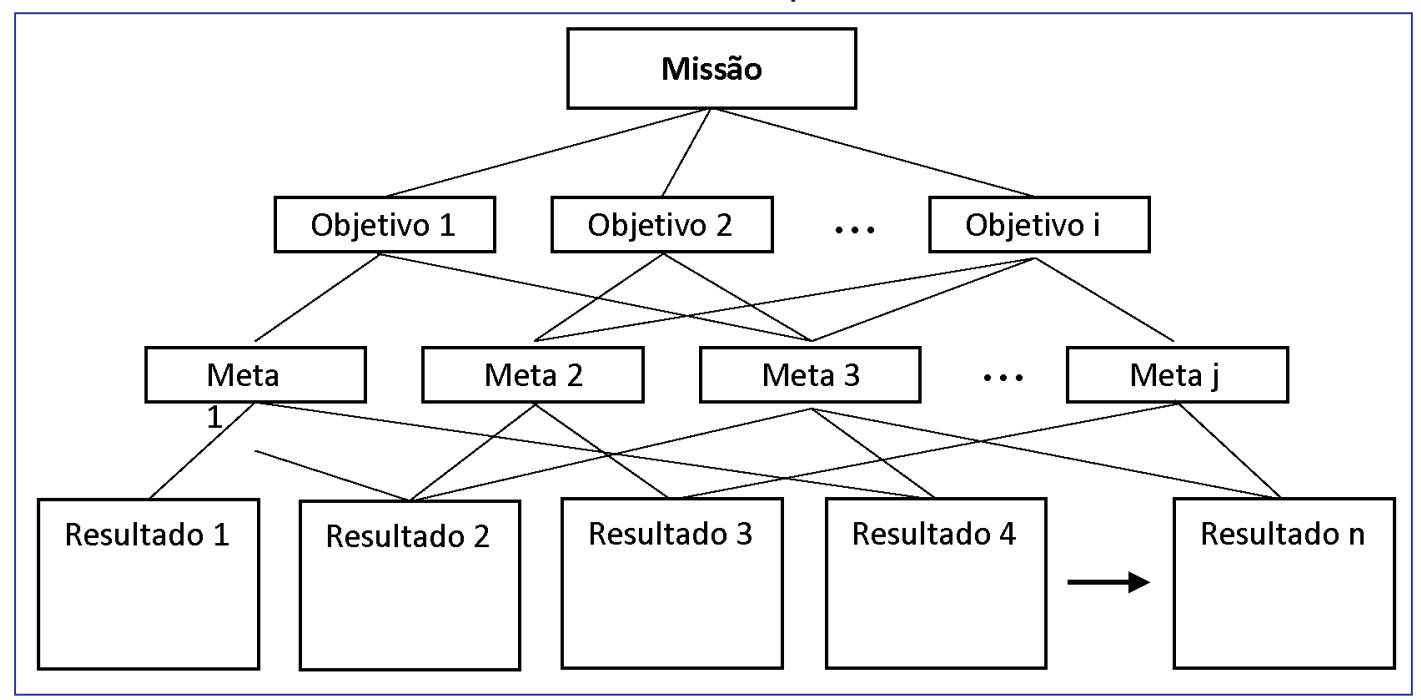

Fonte: Adaptada de Gibson et al. (2019). 


\section{Transferência de conhecimento e tecnologia}

$\mathrm{Na}$ atual sociedade, na qual o conhecimento e a sua aplicação constituem o ativo central, as empresas sentem a necessidade de nova abordagem estratégica (Kandampully, 2002; Jansink, Kwakman \& Streumer, 2005). Essa abordagem trata o conhecimento como fonte essencial de inovação e elemento potencial para criação de vantagem competitiva sustentável (Numprasertchai \& Igel, 2005).

A gestão do conhecimento (GC) é reconhecida por identificar, reunir, organizar, armazenar, compartilhar e aplicar conhecimento, organizando as principais políticas, processos e ferramentas gerenciais e tecnológicas, à luz de uma melhor compreensão dos processos e sua disseminação pela organização (Nonaka, 1994). Sua eficácia requer uma cultura que valorize o conhecimento e as informações, como parte integrante dos processos de negócios das empresas. Fatores críticos de sucesso que propiciam a GC foram identificados no estudo de Akhavan, Hosnavi e Sanjaghi (2009) como sendo gestão de recursos humanos e estrutura organizacional flexível (Cooper, 2001), a ainda uma cultura organizacional propensa (Rego, Pinho, Pedrosa \& Cunha, 2009).

A TC constitui um dos eixos principais da terceira missão da universidade. Permite que os CPUs contribuam para solucionar problemas complexos de vários tipos (Berbegal-Mirabent et al., 2015) com potenciais impactos socioeconômicos. No entanto, pouco se sabe sobre o processo pelo qual o conhecimento científico é transferido dos CPUs para os setores produtivo e social (Chang, Chen \& Fong, 2016). Com a globalização do ensino superior e o suporte tecnológico, a TC se torna mais rápida, frequente e intensa entre universidades e CPUs de diferentes países (Teichler, 2004), propiciando resolução de problemas comuns, influenciando positivamente a mútua colaboração (Veugelers \& Cassiman, 2005). Do ponto de vista das empresas, a TC permite atender às necessidades do mercado, por meio da criação de produtos e tecnologias desenvolvidos por pesquisadores (Franco \& Pinho, 2019). Assim, a colaboração é uma estratégia eficiente para melhorar capacidade de pesquisa, especialmente para unidades de países em desenvolvimento que geralmente possuem recursos limitados (Numprasertchai \& Igel, 2005).

O desafio da colaboração CPU-E parece estar em como reforçar a cadeia de valor de TC em fluxo duplo. Por um lado, aumenta o volume de resultados de pesquisas com potencial para ser incorporado no processo de transferência (papel dos CPUs) e, por outro, gera condições para um aumento da demanda, ou seja, conjunto crescente de empresas e órgãos públicos capazes de se beneficiar com o potencial inovador de conhecimento gerado pelos CPUs (Torres \& Jasso Villazul, 2019).

A TT é o processo de desenvolvimento de aplicações práticas para os resultados de pesquisas científicas (Heinzl, Kor, Orange \& Kaufmann, 2013) relacionado à interação intencional entre pessoas, grupos ou organizações direcionadas ao intercâmbio de tecnologia por diferentes mecanismos (Amesse \& Cohendet, 2001). Associa-se com confiança, proximidade geográfica e políticas universitárias flexíveis para direitos de propriedade intelectual, patentes e licenças (Santoro \& Gopalakrishnan, 2001). Diferentes mecanismos podem ser aplicados nas TT entre CPU-E, de acordo com motivações e recursos disponíveis. Categorizam-se, conforme a interação entre atores, em fluxo unidirecional ou bidirecional. Os principais fatores motivacionais para TT envolvem redução de riscos, como de investimento em P\&D ou de tornar público o conhecimento privado, ou ainda a originalidade da tecnologia (Lee \& Win, 2004).

A TT é um processo complexo que pode seguir diferentes trajetórias, com universidades e empresas participando de diferentes fases, mas que se resumem em dois mecanismos principais.

O primeiro é aquele em que a ciência é uma força motriz de transferência pelo lado das universidades "empurrão científico". Na segunda "atração do mercado", a demanda puxa o processo. No primeiro caso, o conhecimento é transmitido por meio de consultoria, treinamento e atividades de assistência técnica. No segundo, as principais modalidades de transferência são a pesquisa por contrato, a pesquisa por meio de projetos conjuntos e a venda ou licenciamento da propriedade dos resultados da pesquisa gerados nas universidades (Torres \& Jasso Villazul, 2019).

\section{Teorias associadas ao ambiente CPU-E}

Embora não tenha sido localizada uma lente teórica unificada, específica para o ambiente analisado, suas relações e atores, a RSL permitiu identificar as mais recorrentes. Em 78\% dos estudos, os autores não citaram nenhuma teoria. Por outro lado, $22 \%$ indicaram a utilização de, pelo menos, uma teoria.

A lente teórica do capital humano tem sido a mais usada. Diversos autores recorreram a esta teoria para explicar conceitos que envolvam produtividade da pesquisa, colaboração e carreiras de professores em contextos acadêmicos (Berbegal-Mirabent et al., 2015; Boardman, 2009; Boardman \& Bozeman, 2007; Boardman \& Corley, 2008; Boardman \& Gray, 2010; Bozeman, Dietz \& 
Gaughan, 2001; Gaughan \& Corley, 2010; Ponomariov \& Boardman, 2010; Sabharwal \& Hu, 2013; Youtie et al., 2006). Quando pontualmente se analisa o capital humano técnico e científico percebe-se que a abordagem enfatiza a capacidade de pesquisa em nível individual, em termo do conjunto de conhecimento e habilidades científicas, e como ele pode ser afetado por vínculos profissionais e laços de rede. É pertinente se destacar o papel do CPU como sendo projetado para promover o capital humano técnico e científico na universidade (Youtie et al., 2006).

A visão baseada em recursos serviu de lente teórica para os trabalhos de Berbegal-Mirabent et al. (2015), Boardman (2009), Boardman e Gray (2010), Bozeman e Gaughan (2007), Horne et al. (2010), Galán-Muros e Plewa (2016), Santoro e Chakrabarti (1999) e Santoro e Chakrabarti (2001). Reconhece o pacote de recursos heterogêneos (físicos, humanos e organizacionais) como crucial para avanço de tecnologias e para sustentar a vantagem competitiva (Barney, 1991). A colaboração com CPUs pode ser benéfica para empresas de todos os tamanhos. Enquanto empresas maiores geralmente usam relacionamento CPU-E para endereçar questões pré-competitivas e com um horizonte temporal mais amplo, empresas menores também recorrem aos CPUs, pois precisam continuamente desenvolver e comercializar novas tecnologias (geralmente a curto prazo) para entrar em determinados setores ou simplesmente permanecer competitivas em setor existente. Assim, embora as motivações possam variar de acordo com o tamanho da organização, habilidades, conhecimento e acesso às instalações dos CPUs são razões importantes para as colaborações.

Do ponto de vista da teoria institucional, a existência de um sistema de normas (isomorfismo coercitivo) que regule atividades que envolvam atividades como licenciamento, spin-off, TT, TC e patentes balizam as possibilidades de colaborações CPU-E. (Boardman, 2009; Boardman \& Corley, 2008; Boardman \& Ponomariov, 2007; Bruneel et al., 2010).

Por outro lado, lógicas institucionais envolvem princípios básicos de organização pelos quais atores interpretam a realidade organizacional, avaliam alternativas e definem suas identidades e ações. Uma suposição fundamental da perspectiva da lógica institucional diz respeito a diferenças e, portanto, uma importante área de pesquisa trata de teorizar e ilustrar empiricamente essas diferenças globais. Estudos de Thornton (2002) e Lauvås e Steinmo (2019) abordam ambientes CPU-E à luz da coexistência das distintas lógicas institucionais.

Levando-se em consideração as peculiaridades do ambiente analisado, não é de causar estranheza o fato de teorias distintas respaldarem as relações entre os atores. Por vezes, alguns autores empregam múltiplas perspectivas em seus estudos. É fato que há sobreposições entre as visões, mas isto também pode ser entendido como um degrau para se desenvolver uma teoria mais ampla, mesmo que de alcance reduzido, para caracterizar interações CPU-E.

\section{Proposta de framework do ambiente colaborativo}

Como síntese dos resultados da RSL, é possível se propor um framework (Figura 2) envolvendo os atores do ambiente, suas relações assim como o fluxo lógico envolvido. Trata-se de um quadro teórico que congrega os principais elementos apresentados e discutidos durante a pesquisa e que não podem ser negligenciados, principalmente durante o planejamento de um ambiente CPU-E.

Não obstante os interesses próprios de CPUs e organizações externas à universidade que norteiam suas ações, é possível que haja interesses comuns. Esses interesses motivam de um lado CPUs, seus professores, pesquisadores e estudantes guiados pelas suas agendas de pesquisa à luz da teoria do capital humano técnico e científico, e, por outro as organizações externas (empresas, ONGs, associações profissionais e governo) cada qual com sua própria lógica institucional e visão baseada em recursos. Definidas expectativas, níveis de interação, graus de formalização, existência de fluxo financeiro, artefato tecnológicos ou ainda conhecimento codificado, são definidas formas específicas de colaboração sob a óptica da teoria institucional. Seus resultados, traduzidos em benefícios e desvantagens, são medidos em termos de eficácia para a tomada de decisão de se expandir, sustentar ou extinguir os termos de colaboração firmados. Os fluxos de TC e TT se materializam, sejam eles uni/bidirecionais. A essa altura, é possível avaliar o desempenho do CPU como função dos resultados gerados pela colaboração. 
Figura 2

Framework do ambiente colaborativo entre CPU-E

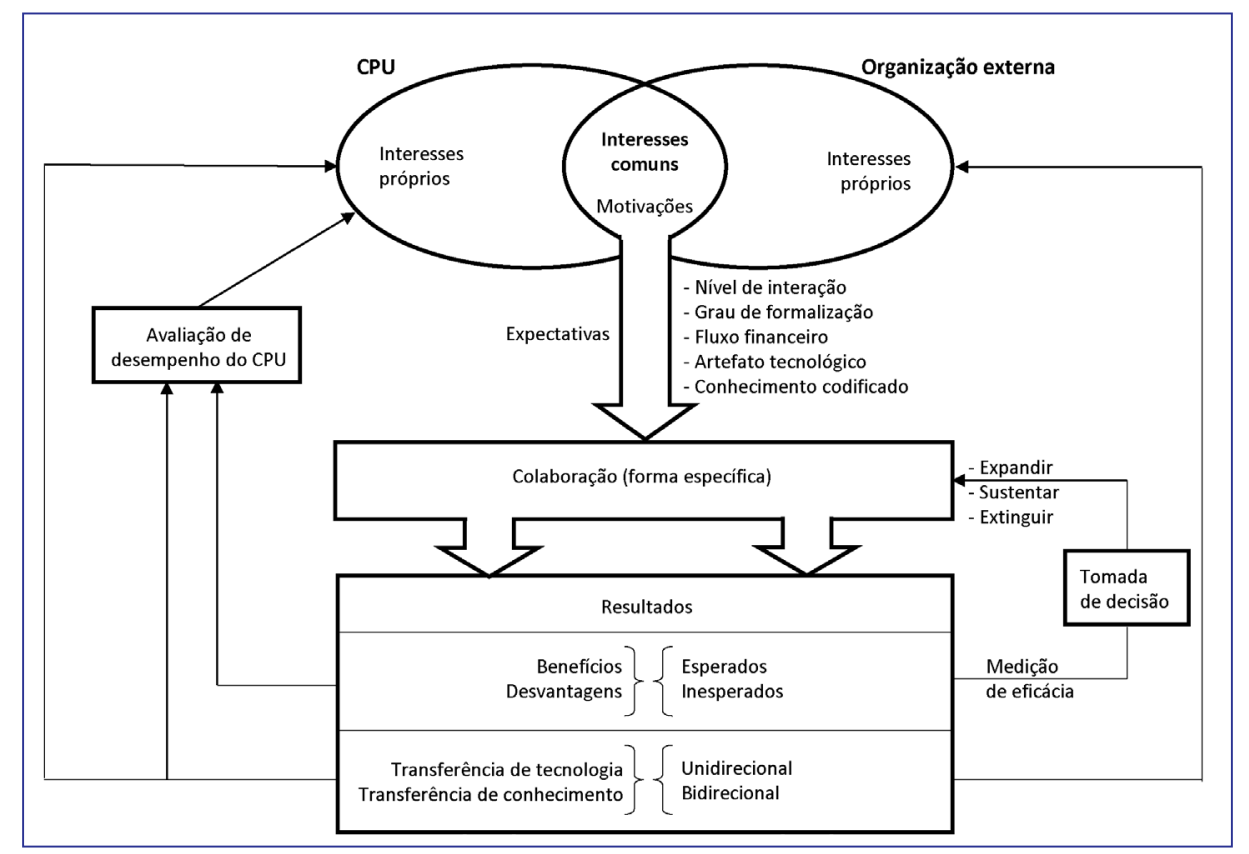

Fonte: Elaborada pelos autores.

\section{CONCLUSÕES}

O estudo realizado indica que a criação de CPUs potencializa a internacionalização dos temas que estão sendo pesquisados em ambientes universitários, fortalece a rede de pesquisadores (professores e estudantes) nacionais e internacionais, aproxima academia, mercado e governo possibilitando a materialização de soluções assinadas em coautoria. Foram selecionados 63 artigos científicos provenientes de busca direta nas bases WoS e Scopus e dos processos de snowballing. Os artigos foram analisados, levando-se em consideração os aspectos: motivação, formas de colaboração, resultados da colaboração, desempenho de CPU, transferências de conhecimento e tecnologia, e as principais teoria abordadas. Por fim, foi elaborado um framework do ambiente colaborativo dos CPUs.

Em termos das principais limitações, a pesquisa realizada nas duas principais bases científicas da área de gestão pode ter levado a desconsiderar artigos publicados em revistas não indexadas, mas que, mesmo assim, sejam relevantes. Além disso, a estratégia de busca foi restrita às publicações em inglês, o que pode ter introduzido um viés a favor de estudos conduzidos em países e instituições que se comuniquem em língua inglesa. No que se refere à estratégia de pesquisa, há que se considerar o caráter seletivo e limitadamente sensível à heterogeneidade social do mundo contemporâneo da RSL, podendo ter causado um viés nos resultados da pesquisa.

Como consequência da RSL, é possível apontar caminhos para realização de investigações adicionais. Como o perfil e as habilidades do pesquisador principal de um CPU, que está na vanguarda da criação do conhecimento, podem influenciar os resultados das colaborações CPU-E visto que, via de regra, esses profissionais são responsáveis tanto pela condução de suas pesquisas, quanto pela gestão dos centros, das equipes e dos projetos associados à CPU. Ainda em relação ao pesquisador principal, é preciso identificar a forma com que entende o papel e a função dos CPUs, enquanto membro, para apoiar seu papel de principal motor propulsor do CPU e gestor das redes internas e externas.

As colaborações CPU-E também podem sugerir o estabelecimento de novos paradigmas metodológicos. Novos métodos de pesquisa podem se estabelecer a partir de práticas emergentes que envolvam os atores e suas características peculiares. De fato, certos métodos podem nascer, fruto da busca por soluções para resolver problemas técnicos colocados pelas empresas, ou ainda serem adaptados pela transferência metodológica entre uma disciplina científica e outra. Essa possível evolução parece ser um dos efeitos indiretos que resultam das interações CPU-E e precisam ser investigadas. 
Como efeito primário das colaborações CPU-E, a literatura analisada menciona o crescimento do estoque de conhecimento das partes envolvidas. A definição do conhecimento enquanto combinação de conhecimento tácito e codificado leva a uma dificuldade para se avaliar esse aumento de estoque de conhecimento em cada um dos colaboradores. Estudos futuros podem endereçar essa questão.

Identificar a relevância de ambientes CPU-E que contêm a participação de estudantes de programas de pós-graduação stricto sensu profissionais para o desenvolvimento de dissertações e teses voltadas para a pesquisa aplicada, resultando em artefatos relevantes para as universidades e para as empresas. As consequências do engajamento acadêmico dos professores e estudantes nas colaborações CPU-E ainda não ficaram evidentes. Os estudos não deixam claro se este envolvimento influencia o processo de ensino-aprendizagem notadamente na produção de valores intangíveis.

É possível analisar a estrutura do ambiente incluindo outros atores com potencial interação com os CPUs, como por exemplo outros CPUs, universidades, ONGs, associações profissionais e instituições públicas. Identificar as motivações que podem levar esses atores a estabelecerem colaborações com CPUs, os fluxos de TC e TT prevalentes, assim como as formas de se avaliar os instrumentos de colaboração.

Nas bases pesquisadas, não foram localizados estudo sobre CPUs no Brasil. Este fato chama a atenção, pois como a literatura descreve, o ambiente de CPU é caracterizado por circunstâncias que promovem ou restringem sua atuação, assim como as colaborações que dele surgem. Aspectos culturais, políticos, sociais, econômicos podem influenciar, potencializar ou restringir o surgimento de CPUs e colaborações com atores externos. A descrição de ambientes CPU-E no Brasil poderá trazer informações relevantes sobre o ambiente e seus atores sendo, inclusive, importante para o poder público do país na formulação de políticas específicas.

\section{AGRADECIMENTOS}

O presente trabalho foi realizado com apoio da Coordenação de Aperfeiçoamento de Pessoal de Nível Superior - Brasil (CAPES). 


\section{REFERÊNCIAS}

Ahn, S. (1995). A new program in cooperative research between academia and industry in Korea, involving Centers of Excellence. Technovation, 15(4), 241-257.

Akhavan, P., Hosnavi, R., \& Sanjaghi, M. E. (2009). Identification of knowledge management critical success factors in Iranian academic research centers. Education, Business and Society: Contemporary Middle Eastern Issues, 2(4), 276-288.

Amesse, F., \& Cohendet, P. (2001). Technology transfer revisited from the perspective of the knowledge- based economy. Research Policy, 30, 1459-1478.

Barney, J. (1991). The resource-based view of strategy: Origins, implications, and prospects. Journal of Management, 7, 97-211.

Berbegal-Mirabent, J., Sánchez-García, J. L., \& Ribeiro-Soriano, D. E. (2015). University-industry partnerships for the provision of R\&D services. Journal of Business Research, 68(7), 1407-1413.

Boardman, P. C. (2009). Government centrality to university-industry interactions: University research centers and the industry involvement of academic researchers. Research Policy, 38(10), 1505-1516.

Boardman, P. C., \& Bozeman, B. (2007). Role Strain in University Research Centers. The Journal of Higher Education, 78(4), 430-463.

Boardman, P. C., \& Corley, E. A. (2008). University research centers and the composition of research collaborations. Research Policy, 37(5), 900-913.

Boardman, P. C., \& Gray, D. (2010). The new science and engineering management: cooperative research centers as government policies, industry strategies, and organizations. The Journal of Technology Transfer, 35, 445-459.

Boardman, P. C., \& Ponomariov, B. L. (2012). Management knowledge and the organization of team science in university research centers. The Journal of Technology Transfer, 39(1), 75-92.

Boardman, P. C., \& Ponomariov, B. L. (2007). Reward Systems and NSF University Research Centers: The Impact of Tenure on University Scientists' Valuation of Applied Commercially Relevant Research. The Journal of Higher Education, 78(1), 51-70.

Bozeman, B., \& Boardman, P. C. (2013). Academic Faculty in University Research Centers: Neither Capitalism's Slaves nor Teaching Fugitives. The Journal of Higher Education, 84(1), 88-120.

Bozeman, B., \& Boardman, P. C. (2003). Managing the New Multipurpose, Multi-discipline University Research Center: Institutional Innovation in the Academic Community. Washington, DC: IBM Endowment for the Business of Government.

Bozeman, B., Dietz, J. S., \& Gaughan, M. (2001). Scientific and technical human capital: an alternative model for research evaluation. International Journal of Technology Management, 22, 716-740.

Bozeman, B., \& Gaughan, M. (2007). Impacts of grants and contracts on academic researchers' interactions with industry. Research Policy, 36, 694-707.

Brereton, P., Kitchenham, B. A., Budgen, D., Turner, M., \& Khalil, M. (2007). Lessons from applying the systematic literature review process within the software engineering domain. Journal of Systems and Software, 80(4), 571-583.

Bruneel, J., D'este, P., \& Salter, A. (2010). Investigating the factors that diminish the barriers to university-industry collaboration. Research Policy, 39, 858-868.

Cameron, K., \& Whetten, D. (1981). Perceptions of organizational effectiveness over organizational life cycles. Administrative Science Quarterly, 26(4), 525-544.

Carayannis, E., Del Giudice, M., \& Della Peruta, M. R. (2014). Managing the intellectual capital within government-university-industry R\&D partnerships, Journal of Intellectual Capital, 15(4), 611-630.

Chang, X., Chen, Q., \& Fong, P. (2016). Scientific disclosure and commercialization mode selection for university technology transfer. Science \& Public Policy, 43, 85-101.

Cooper, D. (2001). Creativity and chaos: preliminary report on the anatomy of research centres/units at higher education institutions in the Western Cape, UWC [University of the Western Cape] Papers in Education, 1, 46-55.

Corona, L. (2015). Patenting in the University and Public Research Centers Considering Their Knowledge Profiles. International Journal of Innovation and Technology Management, 12(3), 1-25.

Edwards, P., Clarke, M., DiGuiseppi, C., Pratap, S., Roberts, I, ... Wentz, R. (2002). Identification of randomized controlled trails in systematic reviews: accuracy and reliability of screening records. Statistic in Medicine, 21(11), 1635-1640.

Etzkowitz, H., \& Kemelgor, K. (1998). The Role of Research Centres in the Collectivisation of Academic Science. Minerva, 36(3), 271-288.

Evans, D., Starbuck, E., Kiresuk, T. J., \& Gee, R. E. (1993). Center for interfacial engineering: an experiment in building industry-university partnerships. International Journal of Technology Management, 8, 622-651.

Felizardo, K. M., Nakagawa, E. Y., Fabbri, S. C. P. F., \& Ferrari, F. C. (2017). Revisão sistemática da literatura em engenharia de software. Rio de Janeiro, RJ: Elsevier.

Franco, M., \& Pinho, C. (2019). A case study about cooperation between University Research Centres: Knowledge transfer perspective. Journal of Innovation \& Knowledge, 4(1), 62-69.

Galán-Muros, V., \& Davey, T. (2017). The UBC ecosystem: putting together a comprehensive framework for university-business cooperation. The Journal of Technology Transfer, 44(4), 1311-1346.

Galán-Muros, V., \& Plewa, C. (2016). What drives and inhibits universitybusiness cooperation in Europe? A comprehensive assessment. R\&D Management, 46(2), 369-382.

Gaughan, M., \& Corley, E. A. (2010). Science faculty at US research universities: The impacts of university research center-affiliation and gender on industrial activities. Technovation, 30(3), 215-222.

Geiger, R. L. (1990). Organized research units: their role in the development of university research. The Journal of Higher Education, 61(1), 1-19. 
Geisler, E., Furino, A., \& Kiresuk, T. J. (1990). Factors in the Success or Failure of Industry-University Cooperative Research Centers. Interfaces, 20(6), 99-109.

Geuna, A., \& Muscio, A. (2009). The governance of university knowledge transfer: A critical review of the literature. Minerva, 47(1), 93-114.

Gibson, E., Daimb, T. U., \& Dabic, M. (2019). Evaluating university industry collaborative research centers. Technological Forecasting \& Social Change, 146, 181-202.

Gray, D. O. (2000). Government-sponsored industry-university cooperative research: an analysis of cooperative research center evaluation approaches. Research Evaluation, 8, 57-67.

Gray, D. O. (2008). Making team science better: Applying improvementoriented evaluation principles to evaluation of research centers. New Dir. Eval., 118, 107-114.

Gray, D. O., \& Steenhuis, H. J. (2003). Quantifying the benefits of participating in an industry university research center: An examination of research cost avoidance Scientometrics, 58(2), 281-300.

Hayton, J. C., Sehili, S., \& Searpello, V. (2010). Why do firms join consortial research centers? An empirical examination of firm, industry and environmental antecedents. Journal of Technology Transfer, 35(5), 494-510.

Heinzl, J., Kor, A. L., Orange, G., \& Kaufmann, H. R. (2013). Technology transfer model for Austrian higher education institutions. The Journal of Technology Transfer, 38, 607-640.

Horne, C. V., Poulin, D., \& Frayret, J. M. (2010). Measuring value in the innovation processes of university-industry research centres. International Journal of Technology, Policy and Management, 10(1/2), 116-136.

Inzelt, A. (2004). The evolution of university-industry-government relationships during transition. Research Policy, 33, 975-995.

Jansink, F., Kwakman, K., \& Streumer, J. (2005). The knowledgeproductive corporate university. Journal of European Industrial Training, 29(1), 40-57.

Kandampully, J. (2002). Innovation as the core competency of a service organization: the role of technology, knowledge and networks. European Journal of Innovation Management, 5(1), 18-26.

Kassab, O., Mutz, R., \& Daniel, H. (2019). Introducing and testing an advanced quantitative methodological approach for the evaluation of research centers: a case study on sustainability science. Research Evaluation, 29(2), 135-149.

Lal, B., Boardman, C., Towery, N. D., Link, J., \& Shipp, S. (2007). Designing the Next Generation of NSF Engineering Research Centers: Insights from Worldwide Practice. Washington, DC: Science and Technology Policy Institute.

Lauvås, T., \& Steinmo, M. (2019). The role of proximity dimensions and mutual commitment in shaping the performance of universityindustry research centres. Innovation, 1-27.

Le Roy, F., Robert, M., \& Lasch, F. (2016). Choosing the best partner for product innovation. International Studies of Management \& Organization, 46(2-3), 136-158.
Lee, D., Kim, S., \& Cha, S. (2014). Evaluating the effectiveness of research centers and institutes in universities: Disciplines and life cycle stages. KEDI Journal of Educational Policy, 11(1), 119-137.

Lee, J., \& Win, H. N. (2004). Technology transfer between university research centers and industry in Singapore. Technovation, 24, 433-442.

Lee, Y. S. (2000). The Sustainability of University-Industry Research Collaboration: An Empirical Assessment. Journal of Technology Transfer, 25, 111-133.

Magro, E., \& Wilson, J. (2013). Complex Innovation Policy Systems: Towards an evaluation mix. Research Policy, 42(9), 1647-1656.

Meyer-Krahmer, F., \& Schmoch, U. (1998). Science-based technologies: university-industry interactions in four fields. Research Policy, 27, 835-851.

Motohashi, K. (2005). University-industry collaborations in Japan: The role of new technology-based firms in transforming the National Innovation System. Research Policy, 34, 583-594.

Nishimura, S. T., Nishimura, E, Goebert, D., Onoye, J. M. M., \& SugimotoMatsuda, J. (2018). A model for evaluating academic research centers: Case study of the Asian/Pacific Islander Youth Violence Prevention Center. Evaluation and Program Planning, 66, 174-182.

Nonaka, I. (1994). A dynamic theory of organizational knowledge creation. Organization Science, 5(1), 14-37.

Numprasertchai, S., \& Igel, B. (2005). Managing knowledge through collaboration: Multiple case studies of managing research in university laboratories in Thailand. Technovation, 25, 1173-1182.

Nursall, A. (2003). Building public knowledge: collaborations between centres, universities and industry. International Journal of Technology Management, 25, 381-389.

Petersen, K., Vakkalanka, S., \& Kuzniarz, L. (2015). Guidelines for conducting systematic mapping studies in software engineering: An update. Information Software Technology, 54(7), 663-685.

Petticrew, M., \& Roberts H. (2006). Systematic Reviews in the Social Sciences: A Practical Guide. Oxford, UK: Blackwell Publishing.

Phillips, D. (1991). New Alliances for Policy and the Conduct of Research and Education, International Journal of Technology Management, 6, 478-487.

Ponomariov, B. L., \& Boardman, P. C. (2010). Influencing scientists' collaboration and productivity patterns through new institutions: University research centers and scientific and technical human capital. Research Policy, 39(5), 613-624.

Rego, A., Pinho, I., Pedrosa, J., \& Cunha, M. P. E. (2009). Barriers and Facilitators to Knowledge Management in University Research Centers. Management Research, 7(1), 33-47.

Roessner, D., Manrique, L., \& Park, J. (2010). The economic impact of engineering research centers: preliminary results of a pilot study. The Journal of Technology Transfer, 35, 475-493.

Rogers, E. M., Hall, B., Hashimoto, M., Steffensen, M., Speakman, K. L. ... Timko, M. K. (1999). Technology Transfer from UniversityBased Research Centers: The University of New Mexico Experience. The Journal of Higher Education, 70(6), 687-705. 
Sá, C. M. (2008). University-Based Research Centers: Characteristics, Organization, Administrative Implications. The Journal of Research Administration, 39(1), 32-40.

Sabharwal, M., \& Hu, Q. (2013). Participation in university-based research centers: Is it helping or hurting researchers? Research Policy, 42, 1301-1311.

Sackett, D. L., Rosenberg, W. M. C., Gray, J. A. M., Haynes, R. B., \& Richardson, W. S. (1996). Evidence based medicine: what it is and what it isn't. BMJ, 1(316), 71-72.

Santoro, M. D., \& Chakrabarti, A. K. (1999). Building industry-- university research centers: some strategic considerations. International Journal of Management Review, 1(3), 225-244.

Santoro, M. D., \& Chakrabarti, A. K. (2001). Corporate strategic objectives for establishing relationships with university research centers. IEEE Transactions on Engineering Management, 48(2), 157-163.

Santoro, M., \& Gopalakrishnan, S. (2001). Relationship Dynamics between University Research Centers and Industrial Firms: Their Impact on Technology Transfer Activities. Journal of Technology Transfer, 26, 163-171.

Scott, A., Steyn, G., Geuna, A., Brusoni, S., \& Steinmuller, E. (2001). The economic returns to basic research and the benefits of universityindustry relationships: a literature review and update of findings (Report for the Office of Science and Technology by SPRU). Brighton, UK: Science and Technology Policy Research.

Stahler, G. J., \& Tash, W. R. (1994). Centers and Institutes in the Research University -- Issues, Problems, and Prospects. Journal of Higher Education, 65(5), 540-554.

Styhre, A., \& Lind, F. (2010). Balancing centripetal and centrifugal forces in the entrepreneurial university: A study of 10 research centres in a technical university. Technology Analysis \& Strategic Management, 22(8), 909-924.

Teichler, U. (2004). The changing debate on internationalisation internationalization of higher education. Higher Education, 48(1), 5-26.
Thornton, P.H. (2002). The rise of the corporation in a craft industry: conflict and conformity in institutional logics, Academy of Management Journal, 45(1), 81-101.

Tidd, J., \& Izumimoto, Y. (2002). Knowledge exchange and learning through international joint ventures: an Anglo-Japanese experience. Technovation, 22(3), 137-145.

Tidd, J., \& Trewhella, M. (1997). Organizational and technological antecedents for knowledge acquisition and learning. R\&D Management, 27(4), 359-375.

Torres, A., \& Jasso Villazul, J. (2019). Capabilities and knowledge transfer: evidence from a university research center in the health area in Mexico. Contaduría y Administración, 64(1), Especial Innovación, 1-16.

Torres Zapata, I. (2019). University Research Centres: Organizational Structures and Performance. Journal of Technology Management \& Innovation, 14(30), 29-42.

Tranfield, D., Denyer, D., \& Smart, P. (2003). Towards a Methodology for Developing Evidence-Informed Management Knowledge by $\mathrm{M}$ eans of Systematic Review. British Journal of Management, 14, 207-222.

Veugelers, R., \& Cassiman, B. (2005). R\&D cooperation between firms and universities. Some empirical evidence from Belgian manufacturing. International Journal of Industrial Organization, 23(5-6), 355-379.

Wohlin, C. (2014). Guidelines for snowballing in systematic literature studies and a replication in software engineering. In Proceedings of 18은 International Conference on Eval and Asses in Soft Engineering, Uxbridge, UK.

Youtie, J., Libaers, D., \& Bozeman, B. (2006). Institutionalization of university research centers: The case of the National Cooperative Program in Infertility Research. Technovation, 26(9), 1055-1063.

José da Assunção Moutinho

ORCID: https://orcid.org/0000-0003-4754-0103

Doutorando e mestre em Gestão de Projetos pela Universidade Nove de Julho (UNINOVE); Gerente de projetos na Universidade do Estado do Rio de Janeiro (UERJ). E-mail: moutinho_pmp@yahoo.com.br

Roque Rabechini Junior

ORCID: https://orcid.org/0000-0002-6277-6571

Doutor em Engenharia de Produção pela Escola Politécnica da Universidade de São Paulo (USP); Professor titular na Universidade Nove de Julho

(UNINOVE). E-mail: rabechinijr@gmail.com 